\title{
Advances in high- $T_{c}$ grain boundary junctions
}

\author{
F. Tafuri ${ }^{1}$, J.R. Kirtley ${ }^{2}$, F. Lombardi ${ }^{3}$, \\ P.G. Medaglia ${ }^{4}$, P. Orgiani ${ }^{4}$, and G. Balestrino ${ }^{4}$ \\ ${ }^{1}$ INFM-Coherentia, Dip. Ingegneria dell'Informazione \\ Seconda Universitá di Napoli, Aversa (CE), Italy \\ ${ }^{2}$ IBM Watson Research Center, Route 134 Yorktown Heights, NY, USA \\ ${ }^{3}$ Department of Microelectronics and Nanoscience, MINA \\ Chalmers University of Technology and Göteborg University, S-41296 Göteborg, Sweden \\ ${ }^{4}$ INFM-Coherentia, Dip. Ingegneria Meccanica, Universitá di Roma Tor Vergata, Roma, Italy \\ E-mail: tafuri@na.infn.it
}

Received February 9, 2004

\begin{abstract}
We review results on two novel types of grain boundary Josephson junctions in the high critical temperature cuprate superconductors. The quality of $\mathrm{YBa}_{2} \mathrm{Cu}_{3} \mathrm{O}_{7-\delta}$ biepitaxial grain boundary Josephson junctions have been improved using a new growth geometry to the point that «tunnel-like» characteristics are reproducibly obtained. The consequent low barrier transmission probabilities are apparently favourable to isolate intrinsic $d$-wave induced effects. We also focus on peculiar aspects of spontaneous currents in HTS Josephson junctions, which are unique and reflect the intimate relation between vortex matter and the Josephson effect. Bicrystal grain boundary Josephson junctions have also been fabricated using artificial superlattice films with as few as six superconducting copper-oxygen planes. Extrinsic and intrinsic $d$-wave effects in these junctions are discussed within the framework of novel designs for $\pi$-circuitry and qubits.
\end{abstract}

PACS: 74.72.-h, 74.50.+r

\section{Introduction}

Ever since the discovery of high critical temperature superconductors (HTS), the fabrication of high quality junctions has presented a difficult materials science task. The goal of producing a tri-layer structure, which could reproduce the very successful achievements of low critical temperature superconductor (S) junctions, with an insulating (I) barrier [1] ( $\mathrm{S}-\mathrm{I}-\mathrm{S})$, was always aimed at, but never really pursued in a systematic and reliable way. This situation reflected the structural complexity of HTS, and the difficulty of finding a good material science recipe for growing a barrier on a highly non-uniform HTS electrode, which was also characterized by poor surface superconducting properties. Recently a significant step in the direction of the goal of an all-HTS trilayer with an insulating barrier was achieved through the structure composed of $\mathrm{La}_{1.85} \mathrm{Sr}_{0.15} \mathrm{CuO}_{4}$ electrodes separated by a one-unitcell-thick $\mathrm{La}_{2} \mathrm{CuO}_{4}$ barrier [2]. The conditions required to fabricate a tri-layer structure are apparently critical and currently limited to a very few special HTS and barrier materials. Significant achievements have been also obtained by alternative junction techniques such as the edge tri-layer $\mathrm{YBCO}-\mathrm{Au}-\mathrm{Nb}$ [3] and the engineered-interface [4] technique.

Despite the slow progress in material science issues related to the realization of HTS junctions, the new physical aspects which have been raised are particularly exciting. What made the novel physics interesting is mostly related to the unconventional order parameter symmetry (OPS) [5-7]. The OPS was made experimentally accessible by reproducible and good quality junctions, such as grain boundary (GB) Josephson junctions (JJs) [8]. The bicrystal technology was fundamental for the development of most of the pioneering experiments on fundamental issues, as well as for simple applications such as SQUIDs. On the other hand, the flexibility of the bicrystal technology, which can provide arbitrary GB orientation, is hindered by the fact that junctions have to be placed basically only on one line (three on a 
quite sophisticated tri-crystal substrate). More recently the biepitaxial technique was revealed to be particularly suitable to study relevant issues of the physics of HTS and HTS Josephson junctions $[9,10]$. The first biepitaxial technique provided $45^{\circ}$ tilt GBs [11]. We extended the same biepitaxial concepts to novel configurations, in which one of the electrodes does not grow along the $c$ axis orientation. Although this geometry appears to be complicated by the presence of an off-axis electrode, it gives major advantages in terms of junction flexibility and junction properties, as demonstrated in this paper. In the present paper we will discuss some important insights into the debate of HTS weak links and $d$-wave induced effects, which have been achieved by using these double angle $\mathrm{CeO}_{2}$-based biepitaxial junctions, and mention novel approaches to HTS junctions based on ultrathin films. What will drive our approach to the problem is the perspective to exploit the «unique» properties of HTS junctions for quantum circuitry, and the need for tunnel-like junctions. This problem clearly involves the fundamental issue of transport in junctions. We will also focus on peculiar aspects of spontaneous currents in HTS JJs, which are unique and reflect the intimate relation between vortex matter and the Josephson effect.

\section{The qubit proposals: need for junctions with tunnel-like properties}

When considering new and exciting physical aspects associated with unconventional OPS and Josephson junctions, the idea of developing $\pi$-circuitry and more in particular novel designs of «quiet qubits» [12-15] is among the most fascinating. The «quiet» aspect of HTS proposals (no need to apply a constant magnetic bias, as opposed to systems based on low temperature superconductor Josephson junctions) probably represents the most relevant feature motivating the interest for HTS qubit systems. Furthermore, the concepts behind the various «qubit» proposals combine several other exciting physical aspects related to $d$-wave OPS, such as Andreev bound states [16,17], time reversal symmetry breaking [18], an imaginary component of the order parameter, and so on.

The «qubit» proposals involving high- $T_{c}$ superconductors basically exploit the fact that JJs with an additional $\pi$ shift in the phase between the electrodes can be produced in the absence of an externally applied supercurrent or field when using superconductors with a $d x^{2}-y^{2}$-wave order parameter symmetry [7]. This may lead to intrinsically doubly degenerate systems, i.e., systems based on JJs with an energy-phase relation with two minima. Current versus the superconducting phase $\left(I_{c}-\varphi\right)$ measurements on $45^{\circ}$ asymmetric and $45^{\circ}$ symmetric bicrystal GB JJs have demonstrated the prevalence of the $2 \varphi$ component in some conditions $[19,20]$. On the other hand, the state of art of HTS JJs and the actual understanding of transport mechanisms in GBs do not presently allow the reproducible determination of the conditions to observe the $2 \varphi$ component. Subsequent investigations raised concerns about the quietness of these devices. It was argued that while on the one hand a $45^{\circ}$ misorientation of the OPS in one electrode is necessary to generate the $\pi$-behavior, it may on the other hand lead to spontaneous currents related to Andreev reflection and more precisely to Andreev bound states $[16,17]$. Such currents may cause additional noise [13] and enhance decoherence mechanisms. This analysis led to the development of alternative designs, in which a five-junction loop (with four ordinary junctions and a $\pi$ junction) takes the place of the original $s$-wave- $d$-wave superconductor junction. In this «macroscopic analog» of [12], the $\pi$ junction removes the need for a constant magnetic bias near $\Phi / 2$.

Contributions to dissipation due to different transport processes, such as channels due to nodal quasiparticles or midgap states or their combination, have been identified and distinguished [21]. In particular cases, decoherence times and quality factors were calculated. It has been also argued that problems in observing quantum effects due to the presence of gapless quasiparticles excitations can be overcome by choosing the proper working phase point [22]. In particular decoherence mechanisms can be reduced by selecting appropriate tunneling directions because of the strong phase dependence of quasiparticle conductance of a $d$-wave GB junction.

The Chalmers group has discussed the dynamics of the junctions in the zero-bias quantum regime and the dependence on junction parameters of the decoherence time in the system [23], determining also the Josephson and the Coulomb energies in submicron junctions. The same group has also experimentally studied the possibility of using size effects in submicron junctions to freeze out low energy quasiparticles, and to induce a prevailing $2 \varphi$ component in the $I_{c}-\varphi$ dependence [20].

These are experimental and theoretical examples of innovative work towards a «quantum» treatment of HTS junctions, and are quite encouraging. Nevertheless a complete «quantum» approach to junction properties and parameters is still missing and typical quantum effects, such as macroscopic quantum tunneling, have not been observed up to now. A lot of work has to be done in this respect covering several different issues, but some «trends» and constraints on junction properties are becoming clear. It is reasonable to expect that for macroscopic quantum tunneling experiments, for instance, it is important to rely on junctions in the tunnel-like limit with relatively high 
values of the normal state resistance. A favourable quantum regime may nevertheless be hindered by the lack of understanding of the junction transport processes, and of the interplay between OPS effects and barrier / microstructure effects. It may be important to reach a regime in which «intrinsic» $d$-wave induced effects are isolated from «extrinsic» effects. Intrinsic effects are only due to the $d$-wave order parameter, while extrinsic effects are mostly due to the $d$-wave order parameter and occur only in junctions with particular morphologies and / or properties. Examples of extrinsic effects are the anomalous dependence of the critical current on the magnetic field [3,24] and the presence of specific spontaneous currents revealed through scanning SQUID microscopy (SSM) [25]. Extrinsic effects may be undesirable and even mask the features of the intrinsic effects. We will discuss in the following Sections how it is possible to reach some regimes of HTS JJs in which intrinsic effects can be isolated.

\section{New biepitaxial technique}

The GB Josephson junctions are obtained at the interface between a (103) $\mathrm{YBa}_{2} \mathrm{Cu}_{3} \mathrm{O}_{7-\delta}$ (YBCO) film grown on a (110) $\mathrm{SrTiO}_{3}$ substrate and a $c$-axis film deposited on a (110) $\mathrm{MgO}$ (Fig.1,a) or $\mathrm{CeO}_{2}$ (Fig.1,b) seed layer. The presence of the $\mathrm{CeO}_{2}$ produces an additional $45^{\circ}$ in-plane rotation of the YBCO axes with respect to the in-plane directions of the substrate $[10,26]$. As a consequence, the GBs are the product of two $45^{\circ}$ rotations, a first one around the $c$ axis, and a second one around the $b$ axis. This configuration produces the desired $45^{\circ}$ misorientation between the two electrodes to enhance $d$-wave OPS effects, as shown in Fig. 1, $d$ and as opposed to the $\mathrm{MgO}$ case of Fig. 1,c, where no in-plane rotation occurs. $\mathrm{MgO}$-based junctions have been proved to be of high quality and their properties to be very weakly influ-

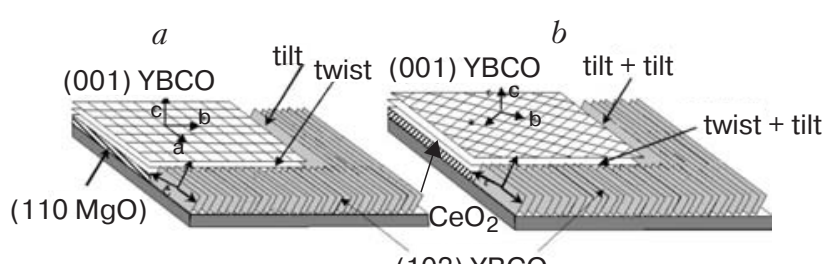

(103) YBCO
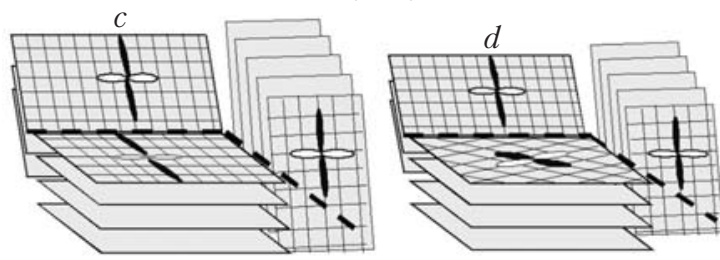

Fig. 1. Sketch of the grain boundary structures. The two limit configurations, tilt and twist, are indicated for junctions based on the $\mathrm{MgO}$ or $\mathrm{CeO}_{2}$ seed layer, respectively. enced by OPS [26]. In this paper MgO based junctions will be discussed mostly for comparison with the $\mathrm{CeO}_{2}$-based junctions.

Furthermore, the degrees of freedom of the fabrication process allow the selection of any possible in-plane orientation for the GB interface. Details about the fabrication process can be found elsewhere $[9,10,26]$. We will define the GB interface angle $\theta$ with respect to the [001] in-plane $\mathrm{SrTiO}_{3}$ direction. In the two limiting configurations, $\theta=0^{\circ}, \theta=90^{\circ}$, the GBs are characterized by a (100) $45^{\circ}$ tilt or twist, respectively, of the $c$ axis with respect to the interface, plus a $45^{\circ}$ tilt around the $c$ axis. In this paper they will be referred to as tilt-tilt and twist-tilt GBs. It is reasonable to assume that these $\mathrm{MgO}$-based and $\mathrm{CeO}_{2}$-based junctions can be considered complementary from the OPS point of view in a circuit design perspective [26].

\section{Transport properties: general features}

The analysis of current versus voltage $(I-V)$ characteristics, while revealing basic properties of the junctions, is a broad topic that goes beyond the aims of this paper. We confine our attention to some specific properties, which are of relevance in our discussion of $d$-wave induced effects in HTS JJs. The general conclusion of different studies realized on the $\mathrm{MgO}$-based and $\mathrm{CeO}_{2}$-based junctions sketched above is that in our GBs, the crucial feature of relatively lower barrier transmission seems to be associated with a $c$-axis tilt. Normal state resistances $R_{N}$ and critical currents $I_{c}$ are the relevant parameters. A direct comparison of the $I-V$ curves for $\mathrm{MgO}$-based and $\mathrm{CeO}_{2}$-based junctions in the extreme tilt and twist limit cases (see Fig. 2) reveals that the barrier transparency may be strongly connected to OPS configuration. In this case the $45^{\circ}$ in-plane rotation of one of the electrodes produces $\pi$-contact behavior. For MgO-based junctions, higher critical current densities and lower normal state resistances characterize the twist limit when compared with the tilt case, reflecting the different grain
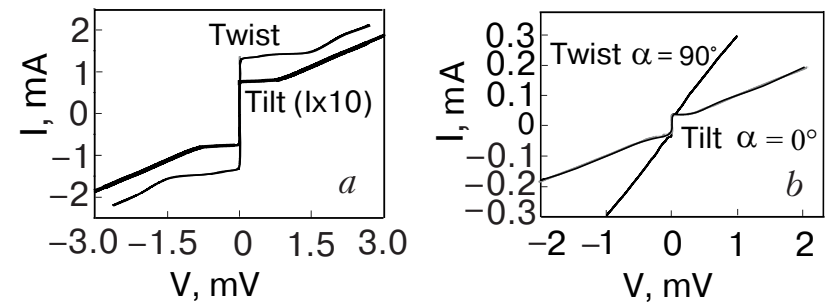

Fig. 2. Current versus voltage $(I-V)$ characteristics of $\mathrm{MgO}$ and $\mathrm{CeO}_{2}$ biepitaxial junctions are reported in the extreme cases of tilt and twist $(T=4.2 \mathrm{~K})$ for junctions of the same width $(10 \mu \mathrm{m})$, respectively. In the $\mathrm{MgO}$ tilt case the current is multiplied by a factor of 10 for clarity. 


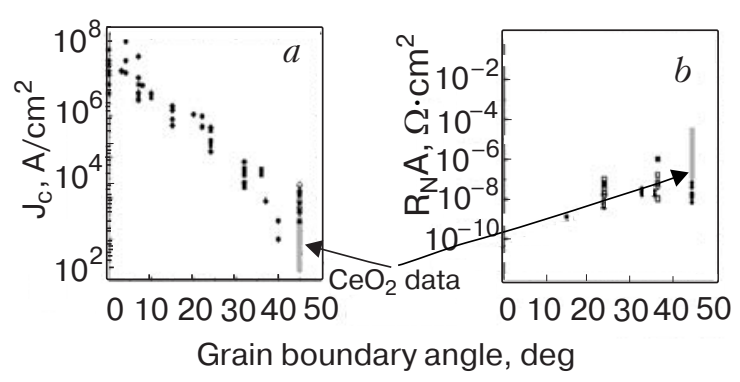

Fig. 3. (a) Critical current densities of $\mathrm{CeO}_{2}$-based junctions of present work (arrows) are compared with data in literature taken from Ref. 8. (b) Normal state resistances of $\mathrm{CeO}_{2}$-based junctions are compared with data in literature taken from Ref. 8.

boundary microstructures. On the other hand, for $\mathrm{CeO}_{2}$-based junctions the influence of the resulting $\pi$-contacts cancels out any difference in critical current densities between the tilt and twist cases. Details will be given in the next Section.

Critical current $I_{c}$ and specific resistivities $R_{N} A$ values (where $A$ is the junction area) are compared with data in literature taken from [8] in Figs. 3, $a$ and $3, b$. The data available in the literature mostly refer to GB junctions where the angle refers to an in-plane tilt. Our grain boundary is more complicated, as discussed in the previous subsection. As a matter of fact, our critical current density $J_{C}$ and $R_{N} A$ values are the lowest and the highest values, respectively, indicating a trend toward tunnel-like behavior. $R_{N} A$ typically range from $10^{-7}$ to $10^{-5} \Omega \cdot \mathrm{cm}^{2}$ at $T=4.2 \mathrm{~K}$, on average at least one order of magnitude higher than the values extracted from measurements on other (in particular bicrystal) types of GB junctions [8]. The $J_{c}$ typically ranges from $10^{2}$ to $5 \cdot 10^{3} \mathrm{~A} / \mathrm{cm}^{2}$.

The tilt and twist configurations are the limiting cases, clearly different from each other (see Fig. 2). A fine tuning of the $I-V$ curves has been clearly observed by changing the interface angle orientations, as shown in Fig. 4, $a$. The tuning of the junction properties follows a very peculiar behavior indicative of $d$-wave OPS (as discussed in the next subsection), while the junction parameters fall in the ranges given above. $I-V$ curves are mostly described by the Resistively Shunted Junction (RSJ) model. We also note in Figs. $4, a, b$ the reproducible presence of hysteretic behavior (switching currents) in $I-V$ characteristics. The hysteretic behavior is mostly observed in the angle range of $\theta \geq 45^{\circ}$ (twist-like regime) and for lower values of $J_{c}$, confirming a general trend towards tunnel-like behavior. The presence of such switching currents may be important for a series of different experiments, such as those aimed to detect macroscopic quantum effects.
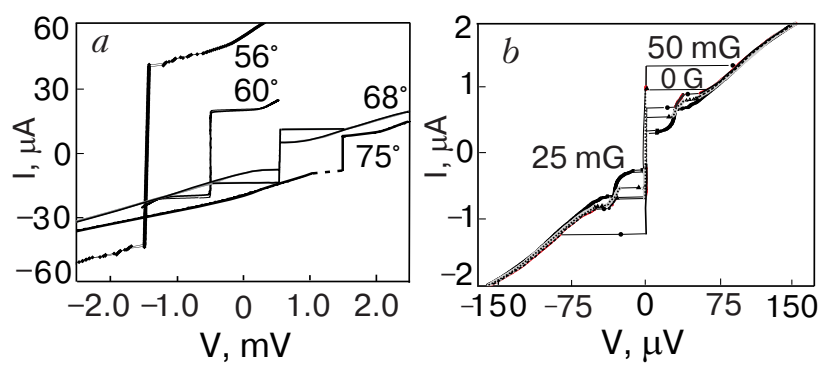

Fig. 4. Current versus voltage characteristics of biepitaxial junctions are reported for various interface orientations measured at $T=4.2 \mathrm{~K}$ in $(a)$ and for a twist junction $\left(90^{\circ}\right)$ for three different values of the magnetic field at $T=0.8 \mathrm{~K}$ in $(b)$, respectively. In $(a)$ curves are shifted along $x$ axis for clarity.

\section{Anisotropy measurements: evidence for $\boldsymbol{d}$-wave induced effects}

In Fig. 5 we report the dependence of $I_{c}$ on the angle $\theta$ for $4 \mu \mathrm{m}$ wide junctions. A clear oscillatory dependence of the critical current $I_{c}$ on $\theta$ was observed [10] as expected in structures dominated by $d$-wave induced effects. This has been observed for various sets of junctions of different widths.

Minima in the critical current are observed for $\theta=0^{\circ}, 34^{\circ}$, and $90^{\circ}$, respectively. These values correspond to configurations in which the tunneling direction (the normal to the barrier) points towards a node of the OP on one of the two sides. The minima at $\theta=0^{\circ}$ and $90^{\circ}$ arise from the position of the nodes in the $c$-axis oriented side of the junction. The minimum at $\theta=34^{\circ}$ occurs when the projection in the $a^{\prime} b^{\prime}$ planes of the normal to the barrier points towards a node of

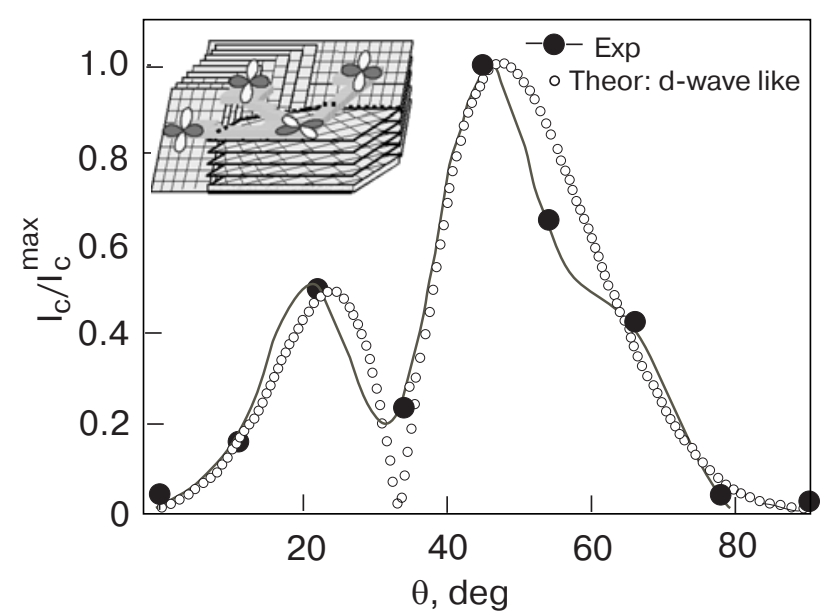

Fig. 5. $I_{C}$ dependence on the angle $\theta$ is reported; experimental data (filled circles) are compared with theoretical

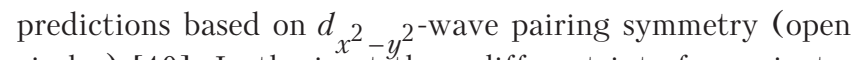
circles) [10]. In the inset three different interface orientations are given as examples. The junction is $4 \mu \mathrm{m}$ wide. 
the OP on the (103) side. In the Sigrist-Rice (SR) phenomenological approach, the Josephson current density of an all $d$-wave junction is given by [5]:

$$
J_{c}=J_{0}\left(n_{x}^{2}-n_{y}^{2}\right)_{L}\left(n_{x}^{2}-n_{y}^{2}\right)_{R} \sin \varphi .
$$

In this expression $J_{0}$ is the maximum Josephson current density, $\varphi$ is the difference between the phase of the OP in the two electrodes, and $n_{x}, n_{y}$ are the projections of the unit vector $\mathbf{n}$ onto the crystallographic axes $x$ and $y$ in the left ( $\mathrm{L}$ ) and right (R) electrode, respectively. Traditionally the SR formula has been applied to junctions where both electrodes are $c$-axis oriented. Specifying the expression above to our noncoplanar configuration $\left(J_{c} \sim \sin 2 \theta\left(2-\cos ^{2} \theta\right)\left(1-3 \sin ^{2} \theta\right) /\left(1+\sin ^{2} \theta\right)\right)$, and assuming a more efficient tunneling in the lobes directions [10], we obtain the curve plotted as open circles in Fig. 5. The experimental behavior is well reproduced by the SR-like theoretical prediction. The $I_{c}(\theta)$ dependence in agreement with the SR formula apparently seems to be mostly determined by the OPS.

We believe that our junction configuration, with low barrier transmission probabilities, preserves the directionality of the Cooper pairs, whether the transport be by tunneling or some other mechanism. Therefore it is in principle more sensitive to the angular dependence of the order parameter symmetry and therefore reveals $d$-wave induced behavior. The anisotropy measurements have demonstrated for the first time that «intrinsic» $d$-wave effects are dominant in the phenomenology of the Josephson junctions themselves (not inserted in any loop) independently of the interface details [10]. The good agreement with the SR formula apparently suggests that the $I_{C}(\theta)$ dependence is mostly determined by the OPS. In this case the grain boundary microstructure, which is the other main effect ruling the junctions' properties, does not significantly contribute to the qualitative behavior of $I_{c}(\theta)$. In other words, deviations from the Sigrist-Rice behavior may indicate a more significant contribution played by the actual GB microstructure to the angular dependence of $I_{c}$. The GB microstructure definitely contributes to form a «tunnel-like» barrier, characterized by higher values of the normal state resistance.

\section{Scanning SQUID microscopy and spontaneous currents}

In this Section and the next we will deal with some aspects related to vortex matter and the Josephson effect, and in particular to the presence of spontaneous currents (i.e., spontaneous magnetization with random orientation and the half flux quantum effect) in $\mathrm{CeO}_{2}$-based biepitaxial junctions. Phenomena re- lated to spontaneous currents are peculiar to HTS Josephson junctions and $\pi$-contacts systems. Scanning SQUID Microscopy [25] is an appropriate technique to image and study the magnetic response of different samples, and can be used in appropriate configurations to study phenomena in zero field cooling, and therefore spontaneous currents. Spontaneous currents may have different origins depending on the system where they are observed. The half flux quantum effect (such as observed in the tri-crystal experiments) was the first remarkable example of spontaneous currents [7]. In this case the different flux distributions depend only on the intentionally introduced sample geometry and on the typical scaling lengths of the junctions involved. SSM has also demonstrated the presence of randomly distributed spontaneous currents along GBs [24]. These are determined by naturally occuring $\pi$-loops due to a combination of the $d$-wave OPS and the junction morphology (facetting) $[24,27,28]$.

\section{Spontaneous magnetization with random orientation}

We first address spontaneous currents due to $\pi$-loops and how it is possible to prevent their formation [28]. To this aim we investigated through SSM the magnetic behavior of grain boundaries of biepitaxial samples cooled in zero field and characterized by different junction parameters.
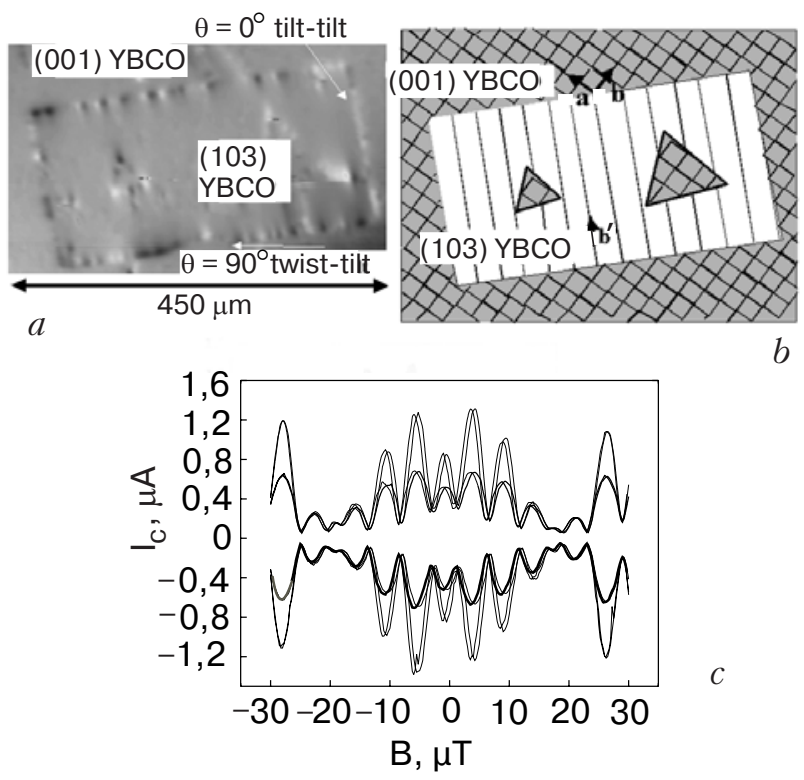

Fig. 6. (a) SSM image of a $600 \times 400 \mu \mathrm{m}$ area. The GBs are marked by the presence of spontaneous currents. The sample was cooled and imaged at $T=4.2 \mathrm{~K}$ in nominally zero field. (b) Sketch of the grain boundaries imaged in (a). (c) Magnetic pattern of an hysteretic twist biepitaxial junction measured at $T=4.2 \mathrm{~K}$. The two different curves refer to the critical and the retrapping currents. The junction is $20 \mu \mathrm{m}$ wide. 
In Fig. 6, $a$ we give evidence of the presence of spontaneous currents along the GBs in the tilt, twist, and intermediate situations represented for instance by the sides of the photolithographically defined triangles sketched in Fig. 6,b. This behavior is consistent with the presence of $\pi$-loops along the GBs and with expectations based on faceted interfaces, in agreement with other experiments on bicrystal [24] and biepitaxial junctions [27]. The noise associated with such currents, due to $\pi$-loops along GBs, provide a simple example of how HTS JJs may be intrinsically noisy. Such a behavior is associated with samples characterized by higher critical current densities $J_{c}$ [29]. In the literature it has been shown that the presence of $\pi$-loops is accompanied by an anomalous magnetic pattern [3,24] such as the one reported in Fig. 6,c measured on one of our junctions. In this case the $I-V$ characteristic is hysteretic and we report both the critical escape and retrapping currents.

In Fig. 7, where a scanning SQUID microscope image of a $800 \times 800 \mu \mathrm{m}$ area is reported, no spontaneous magnetization was detected along any of the GB interfaces, which had arbitrary orientation [28]. Single flux quantum Josephson vortices are randomly present along the GBs. In this case we have changed $J_{c}$ and as a consequence the Josephson penetration depth. This has been done by reducing the thickness of the film in Fig. 7 to about one third of the thickness of the sample reported in Fig. 6, $a$. Differences in the $J_{c}$ values correspond to the general feature in HTS JJs, that $J_{c}$ does not perfectly scale with the film thickness [8].

If we try to interpret these results, keeping in mind the $d$-wave nature of these junctions, as revealed by the anisotropy measurements, we attribute the absence of any spontaneous magnetization to the locally reduced $J_{c}$. This experimental conclusion is consistent

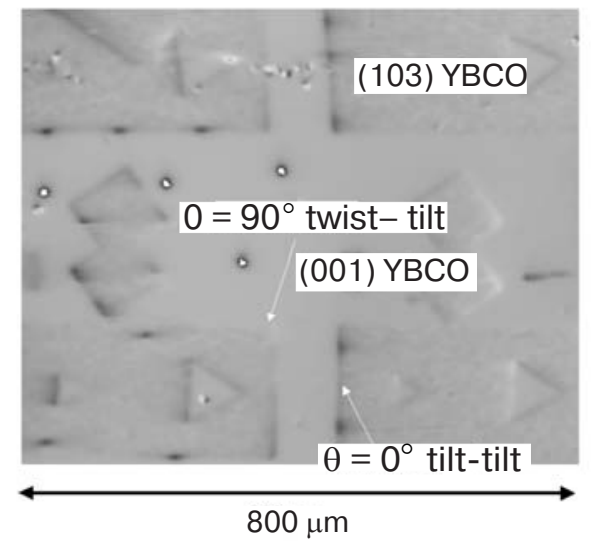

Fig. 7. SSM image of an $800 \times 800 \mu \mathrm{m}$ area, enclosing tilt-tilt and twist-tilt GBs. The sample was cooled in nominally zero field and imaged at $T=4.2 \mathrm{~K}$. No randomly oriented spontaneous magnetization was detected along the GB interfaces. with numerical solutions of the Sine-Gordon equation for a $0-\pi$ Josephson junction [30-32]. In this modeling the spontaneous magnetization decreases when the ratio of the Josephson penetration depth $\lambda_{J}$ to the facet spacing becomes large, as discussed in detail in [28,31]. We have modeled this for a regular array, varying the facet width $L_{\pi}$. For the facetted configuration there is a threshold to observe spontaneous magnetization $\left(L_{\pi} \simeq \lambda_{J}\right)$ [28]. Sufficiently long penetration depths made measurements of the Josephson critical currents as a function of the misorientation angle insensitive to facetting effects, and represent the crucial feature in preventing the formation of spontaneous currents due to $\pi$-loops in the systems analyzed.

We recall another interesting related configuration in which spontaneous currents with random orientation have been observed [33]. This case enriches possible scenarios of spontaneous currents. We refer to $c$-axis YBCO thin films grown on a $\mathrm{MgO}$ seed layer on a (110) $\mathrm{SrTiO}_{3}$ substrate (which basically represents the $c$-axis electrode of the $\mathrm{MgO}$ based biepitaxial configuration). Spontaneous currents were observed to be spatially correlated with impurities due to the presence of the so-called green phase due to $\mathrm{Y}$ excess in $c$-axis films. The origin of such currents is unclear. It may be due to time reversal symmetry breaking (BTRS) or due to the spontaneous nucleation of topological defects in phase transitions (for instance, the pinning of a vortex tangle, produced near $T_{c}$ in a Berezinskii-Kosterlitz-Thouless [34] type transition in the nearly two dimensional superconductor YBCO). BTRS may be related to an imaginary component of the OPS near a surface facing the insulating green phase [18]. This would in principle be the only configuration able to prove the origin of BTRS as due to an imaginary component of the order parameter, since in junction interfaces the same effect could also be due simply to Andreev bound states $[35,36]$. The lack of an experiment showing reproducible, well defined isolated spontaneous currents in controlled systems is a limitation for the former interpretation, and related arguments on fractional vortices. More recent studies on phase transitions on amorphous superconductor $\mathrm{Mo}_{3} \mathrm{Si}$ in ring geometry make the latter possibility feasible [37]. Further investigations are required for a definitive answer.

\section{Half flux quantum}

Once the conditions which lead to the absence of spontaneous currents due to $\pi$-loops along GBs were determined, we studied if and how the well known half flux quantum effect occurs in our peculiar junction configurations. 


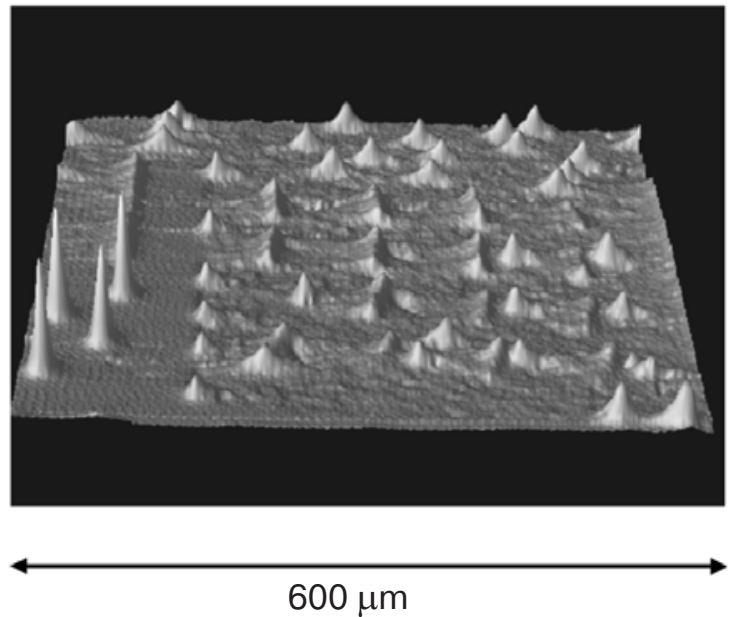

Fig. 8. SSM image of a $600 \times 600 \mu \mathrm{m}$ area, enclosing different types of Gbs. The sample was cooled in a small magnetic field and imaged at $T=4.2 \mathrm{~K}$. Flux tubes appear randomly localized in bulk material and along grain boundaries. Magnetic flux also appears along some grain boundary lines.

Figure 8 is a 3-dimensional SSM image of a large area of a biepitaxial sample containing different interfaces and types of grain boundary, cooled in presence of a small magnetic field. Various types of vortices and spread flux can be observed. We notice on the left, four standard in-plane Abrikosov vortices characterized by flux well localized in a narrow area. On the top anisotropic vortices in the (103) part of the YBCO film are also visible. These are more spread than the in-plane Abrikosov vortices. Both these types of vortices are a useful reference to determine the flux present in the center of the image along the lithographically pre-determined contours (triangles in particular). In contrast with the ordinary vortices, the flux along the triangles will not vanish even in zero field cooling. This is due to the particular triangular shapes (defined by grain boundaries with different orientations) which were designed in order to observe the half flux quantum effect.

A schematic of a section of the sample imaged in Fig. 8, a set of 16 isosceles triangles (triangle side $L=$ $=50 \mu \mathrm{m})$, is shown in Fig. 9, $a$. Each triangle is rotated clockwise by 3 degrees from the last, moving from bottom to top along the rows of triangles, and from left to right along the columns. Corners which have a sign change in the product of the normal components of the gap functions on opposite sides of the grain boundaries are expected to have spontaneously generated Josephson vortices with half of the superconducting flux quantum of flux localized at them, if the Josephson depth is short relative to the length of a side of the triangle. In this design the half flux quantum vortices should systematically shift to different corners as the triangle orientation is changed. Figure $9, b$ shows the corresponding SSM image of the $a$
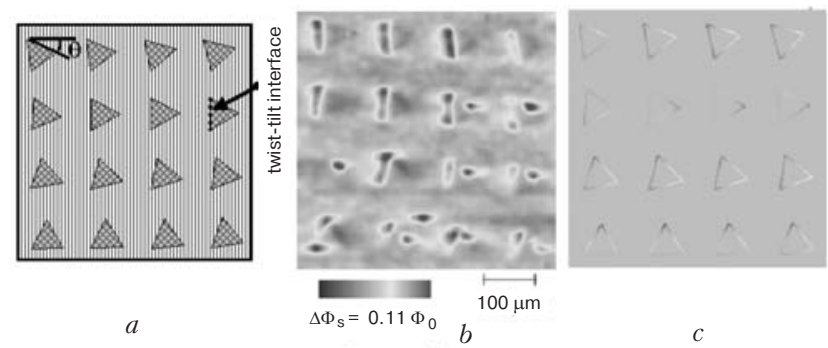

Fig. 9. (a) Schematic diagram of the sample geometry. (b) SSM image of a $450 \times 450 \mu \mathrm{m}$ area, enclosing a set of 16 triangles with various interface orientations. The image was taken at $T=4.2 \mathrm{~K}$. (c) Simulated spontaneous fluxes along the triangle interfaces, assuming $d$-wave momentum dependences of the pairing wavefunction.

sample cooled and imaged in a magnetic field such that there is approximately 1 flux quantum $\left(\Phi_{0}\right)$ trapped per triangle. This image is the two-dimensional view of the central part of the image of Fig. 8. Spontaneous (since it was observed also when the sample was cooled in zero field) magnetization is visible along some interfaces and at some corners, and a systematic behavior clearly appears moving from the top row where the flux is spread along GBs down to the bottom where the flux is more localized in some corners, respectively.

The fact that in some circumstances the half flux quantum can be spread along the grain boundaries rather than being concentrated in the corner, is consistent with long Josephson penetration depths and low barrier transmissions [38], occurring in our junctions. For comparison, simulations of the expected spontaneous currents in our triangular grain boundaries are shown in Fig. 9,c. These simulations numerically solve the Sine-Gordon equation for a facetted grain boundary [31], with the sign and magnitude of the Josephson critical current density along the grain boundaries chosen using the Sigrist-Rice expressions [5], assuming the standard $d_{x^{2}-y^{2}}$-like pairing symmetry [28]. It was assumed there was a total of one $\Phi_{0}$ of magnetic flux in each triangle. The Josephson penetration depth $\lambda_{J}$ was set equal to $\lambda_{J 0} / \sqrt{\left|j_{c}(\theta)\right|}$, where $j_{c}(\theta)$ contains the dependence of the critical current on grain boundary geometry. For the simulations $\lambda_{J}(0)$ was assumed to be 5 microns. Details about the simulations can be found in [28]. Apart from minor local deviations, there is qualitative agreement between experimental data and the modeling.

\section{Discussion: extrinsic and intrinsic $d$-wave effects in grain boundary junctions}

The features discussed in the previous Section prove the existence of «pure» intrinsic $d$-wave effects in GB 
JJs, as indicated in anisotropy transport measurements and the half flux quantum effect, in agreement with theoretical expectations. Moreover experimental conditions can be chosen in which spontaneous extrinsic currents may be substantially reduced. It is possible therefore in the biepitaxial technology to observe the half flux quantum effect and to suppress spontaneous magnetization with random orientations. In other words there seems to be an accessible scaling range of junction parameters to reach a favourable «regime» where $d$-wave induced intrinsic features are very robust, and extrinsic features such as noise from $\pi$-loops can be severely limited. The coherence related to the OPS seems to be preserved on a scale larger than the faceting and/or any interface impurity characteristic lengths. Both from anisotropy tranport measurements and SSM analysis, results on junctions can be reasonably explained without invoking a significant imaginary component of the OPS $[18,35]$.

A study based on radio-frequency and SSM measurements [39] on the same tilt-tilt samples seemed to show the absence of the type of spontaneous magnetization reported, for instance, in Fig. 7, and Andreev bound states are effects which can co-exist. This also means that the possibility of preventing the spontaneous currents discussed above applies only to those generated by $\pi$-loops. However, a threshold of 0.01 of the flux quantum $\Phi_{0}$ for other additional mechanisms generating noise in HTS JJs is set by SSM measurements. The tunnel-like behavior inferred from different experiments is the other crucial feature of these biepitaxial junctions; it favours a promising regime where junction quantum effects could manifest. The additional degree of freedom offered by the biepitaxial technique, to vary the interface orientation, can be crucial to find more precise conditions in which to optimize specific performances and even to reduce decoherence mechanisms [22]. For instance, in the range $50^{\circ} \leq \theta \leq 80^{\circ}$ we could obtain a regime where $I-V$ curves are hysteretic and the midgap states effect should be reduced.

\section{Josephson junctions based on ultrathin CBCO films}

In this Section we discuss a «novel» type of HTS JJs. The philosophy is to develop controlled systems which may give in the proper limit more uniform barrier interfaces. This is realized by exploiting GB junctions and ultrathin films, i.e., artificially layered HTS [40], such as $\left[\mathrm{Ba}_{0.9} \mathrm{Nd}_{0.1} \mathrm{CuO}_{2+x}\right]_{m} /\left[\mathrm{CaCuO}_{2}\right]_{n}$ $(\mathrm{CBCCO}-m \times n)$. These films (similarly to all existing HTS cuprates) are composed by a stacking sequence of two structural subunits having different functions, namely the charge reservoir (CR) block

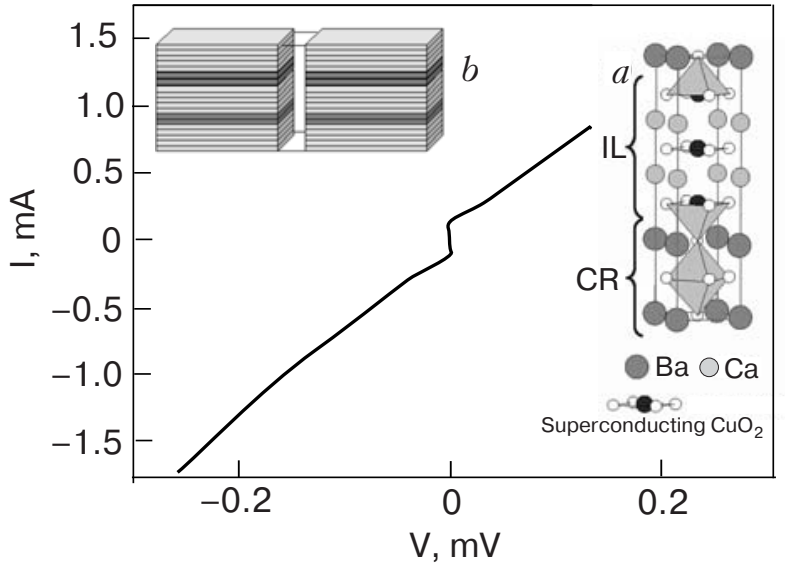

Fig. 10. $I-V$ curve in zero field for a $1 \mathrm{~mm}$ wide bicrysal junction. The misorientation angle is in this case $24^{\circ}$ and the configuration is asymmetric. The typical stacking sequence of the charge reservoir (CR) block and the infinite layer (IL) superconducting block characteristic of HTS compounds and of the CBCO compound used in this experiment is reported in inset $(a)$. The grain boundary schematic diagram is shown in inset $(b)$ : only 6 superconducting $\mathrm{CuO}_{2}$ planes are present on each electrode; Josephson phenomena should be associated only with such planes.

and the infinite layer (IL) superconducting block (see inset a Fig. 10). The IL block always consists of $\mathrm{CuO}_{2}$ planes separated by an alkaline earth (mostly $\mathrm{Ca}$ ) plane, while the structure and the chemical composition of the CR block vary from compound to compound. The structural and transport properties of these compounds have been discussed elsewhere $[40,41]$. We have for the first time realized Josephson junctions composed of only a few superconducting $\mathrm{CuO}_{2}$ planes (6 layers in particular). For this experiment we have focused in particular on ultrathin $\left[\mathrm{Ba}_{0.9} \mathrm{Nd}_{0.1} \mathrm{CuO}_{2+x}\right]_{5} /\left[\mathrm{CaCuO}_{2}\right]_{2} /\left[\mathrm{Ba}_{0.9} \mathrm{Nd}_{0.1} \mathrm{CuO}_{2+x}\right]_{5}$ $/\left[\mathrm{CaCuO}_{2}\right]_{2} /\left[\mathrm{Ba}_{0.9} \mathrm{Nd}_{0.1} \mathrm{CuO}_{2+x}\right]_{5}(5 / 2 / 5 / 2 / 5)$ structures and have employed the bicrystal technique. The junction schematic diagram is shown in the inset (b) of Fig. 10, where the six superconducting $\mathrm{CuO}_{2}$ planes are shown. The CBCO film is only $8 \mathrm{~nm}$ thick.

The current-voltage $I-V$ curves reported in Fig. 10 correspond to a $w=1 \mathrm{~mm}$ wide bicrystal junction measured at $T=4.2 \mathrm{~K}$. The misorientation angle is in this case $24^{\circ}$ and the configuration is asymmetric. Similar behavior was observed for symmetric junctions up to 5 $\mathrm{mm}$ wide. The shape is RSJ-like and $I-V$ were modulated by the magnetic field. Details will be discussed elsewhere.

The simple structure of the grain boundary composed by 6 superconducting $\mathrm{CuO}_{2}$ layers (due to the highly controlled structure of the $5 / 2 / 5 / 2 / 5$ artificial structure) also allows a reliable estimation of the 
coupling along the $a b$ planes of two $\mathrm{CuO}_{2}$ layers separated by a $24^{\circ}$ asymmetric or symmetric GB: in particular we calculate a critical current density per plane of about $(0.2-0.3) \cdot 10^{2} \mathrm{~A} / \mathrm{cm}^{2}$.

Additional evidence of the Josephson behavior comes from the observation through SSM of Josephson vortices, which will be discussed elsewhere. In this case the magnetic fields associated with the Josephson vortices are influenced by the extremely large Pearl lengths of these very thin films.

\section{Conclusions}

We have discussed different issues of grain boundary HTS Josephson junctions. To produce high quality junctions is a crucial step to pursue quantum circuitry based on HTS and to investigate reliably all the exciting phenomenology occurring in HTS junctions. Good quality «tunnel-like» Josephson junctions can be fabricated by exploiting the biepitaxial technique. The anisotropic transport measurements and the half flux quantum effect, both in agreement with theoretical expectations, prove the existence of «pure» intrinsic $d$-wave effects in GB JJs. If this is combined with the absence of spontaneous magnetization in situations where facetting could be expected to be an issue, there seems to be an accessible scaling range of junction parameters to reach a favorable «regime» where $d$-wave induced intrinsic features are very robust and dominant over extrinsic features such as noise from $\pi$-loops. A remarkable feature is the presence of Josephson phenomena in nano-structured systems (ultrathin films) along macroscopic dimensions of the order of a few millimeters. More specifically we are able to identify Josephson phenomena in films with 6 superconducting layers in a nominally very uniform configuration. No analogous situation exists for other HTS junctions with thicker electrodes. This more controlled system could open some perspectives also for further understanding of the transport mechanisms in grain boundary junctions.

This work has been partially supported by the ESF projects «ח-Shift» and «QUACS». The authors would like to thank A. Barone, T. Bauch, S. Kubatkin, K. Moler, and C. Tsuei for useful discussions.

1. A. Barone and G. Paternó, Physics and Applications of the Josephson Effect, J. Wiley, New York (1982).

2. I. Bozovic, G. Logvenov, M.A.J. Verhoeven, P. Caputo, E. Goldobin, and T.H. Geballe, Nature 422, 873 (2003).

3. H.J.H. Smilde, Ariando, D.H.A. Blank, G.J. Gerritsma, H. Hilgenkamp, and H. Rogalla, Phys. Rev. Lett. 88, 057004 (2002); H. Hilgenkamp, Ariando, H.J.H. Smilde,
D.H.A. Blank, G. Rijnders, H. Rogalla, J.R. Kirtley, and C.C. Tsuei, Nature 422, 50 (2003).

4. B.H. Moeckly and K. Char, Appl. Phys. Lett. 71, 2526 (1997).

5. M. Sigrist and T.M. Rice, J. Phys. Soc. Jpn. 61, 4283 (1992); M. Sigrist and T.M. Rice, Rev. Mod. Phys. 67, 503 (1995).

6. D.J. van Harlingen, Rev. Mod. Phys. 67, 515 (1995).

7. C.C. Tsuei and J.R. Kirtley, Rev. Mod. Phys. 72, 969 (2000) (and references therein).

8. H. Hilgenkamp and J. Mannhart, Rev. Mod. Phys. 74, 485 (2002) (and references therein).

9. F. Tafuri, F. Miletto Granozio, F. Carillo, A. Di Chiara, K. Verbist, and G. Van Tendeloo, Phys. Rev. B59, 11523 (1999).

10. F. Lombardi, F. Tafuri, F. Ricci, F. Miletto Granozio, A. Barone, G. Testa, E. Sarnelli, J.R. Kirtley, and C.C. Tsuei, Phys. Rev. Lett. 89, 207001 (2002).

11. K. Char, M.S. Colclough, S.M. Garrison, N. Newman, and G. Zaharchuk, Appl. Phys. Lett. 59, 733 (1991).

12. L.B. Ioffe, V.B. Geshkenbein, M.V. Feigel'man, A.L. Fauchere, and G. Blatter, Nature 398, 679 (1999).

13. G. Blatter, V.B. Geshkenbein, and L.B. Ioffe, Phys. Rev. B63, 174511 (2001).

14. A. Blais and A.M. Zagoskin, Phys. Rev. A61, 42308 (2000); A.M. Zagoskin, cond.-mat. 9903170.

15. Y. Makhlin, G. Schon, and A. Shnirman, Rev. Mod. Phys. 73, 357 (2001).

16. C.-R. Hu, Phys. Rev. Lett. 72, 1526 (1994).

17. T. Lofwander, V. Shumeiko, and G. Wendin, Supercond. Sci. Tech. 14, R53 (2001) (and references therein).

18. M. Sigrist, Progr. Theor. Phys. 99, 899 (1998).

19. E. Ilichev, V. Zakosarenko, R.P.J. IJsselsteijn, H.E. Hoenig, V. Schultze, H.G. Meyer, M. Grajcar, and R. Hlubina, Phys. Rev. B60, 3096 (1999); E. Ilichev, M. Grajcar, R. Hlubina, R.P.J. IJsselsteijn, H.E. Hoenig, H.G. Meyer, A. Golubov, M.H.S. Amin, A.M. Zagoskin, A.N. Omelyanchuk, and M.Yu. Kupriyanov, Phys. Rev. Lett. 86, 5369 (2001).

20. T. Lindstrom, S.A. Charlebois, A.Ya. Tzalenchuk, Z. Ivanov, M.H.S. Amin, and A.M. Zagoskin, Phys. Rev. Lett. 90, 117002 (2003).

21. Ya.V. Fominov, A.A. Golubov, and M.Yu. Kypryanov JETP Lett. 77, 587 (2003).

22. M.H.S. Amin and A. Smirnov, Phys. Rev. Lett. 92, 17001 (2004).

23. A.Ya. Tzalenchuk, T. Lindstrom, S.A. Charlebois, E.A. Stepantsov, Z. Ivanov, and A.M. Zagoskin, Phys. Rev. B68, 1005001 (2003).

24. J. Mannhart, H. Hilgenkamp, B. Mayer, Ch. Gerber, J.R. Kirtley, K.A. Moler, and M. Sigrist, Phys. Rev. Lett. 77, 2782 (1996); H. Hilgenkamp, J. Mannhart, and B. Mayer, Phys. Rev. B53, 14586 (1996).

25. J.R. Kirtley, M.B. Ketchen, K.G. Stawiasz, J.Z. Sun, W.J. Gallagher, S.H. Blanton, and S.J. Wind, Appl. Phys. Lett. 66, 1138(1995) (and references therein).

26. F. Tafuri, F. Carillo, F. Lombardi, F. Miletto Granozio, F. Ricci, U. Scotti di Uccio, A. Barone, G. Testa, E. Sarnelli, and J.R. Kirtley, Phys. Rev. B62, 14431 (2000). 
27. J.R. Kirtley, P. Chaudhari, M.B. Ketchen, N. Khare, Shawn-Yu Lin, and T. Shaw, Phys. Rev. B51, R12057 (1995).

28. F. Tafuri, J.R. Kirtley, F. Lombardi, and F. Miletto Granozio, Phys. Rev. B67, 174516 (2003).

29. R.G. Mints, Ilya Papiashvili, J.R. Kirtley, H. Hilgenkamp, G. Hammerl, and J. Mannhart, Phys. Rev. Lett. 89, 67004 (2002); R.G. Mints and Ilya Papiashvili, Phys. Rev. B64, 134501 (2001).

30. C.S. Owen and D.J. Scalapino, Phys. Rev. 164, 538 (1967).

31. J.R. Kirtley, K.A. Moler, and D.J. Scalapino, Phys. Rev. B56, 886 (1997).

32. E. Goldobin, D. Koelle, and R. Kleiner, Phys. Rev. B67, 224515 (2003).

33. F. Tafuri and J.R. Kirtley, Phys. Rev. B62, 13934 (2000).

34. J.M. Kosterlitz and D.J. Thouless, J. Phys. C6, 1181 (1973).

35. M.H.S. Amin, A.N. Omelyanchouk, and A.M. Zagoskin, Phys. Rev. B63, 212502 (2001); M.H.S. Amin,
S.N. Rashkeev, M. Coury, A.N. Omelyanchouk, and A.M. Zagoskin, Phys. Rev. B66, 174515 (2002).

36. T. Lofwander, V.S. Shumeiko, and G. Wendin, Phys. Rev. B62, R14653 (2003).

37. J.R. Kirtley, C.C. Tsuei, and F. Tafuri, Phys. Rev. Lett. 90, 257001 (2003).

38. C.C. Tsuei and J.R. Kirtley, Phys. Rev. Lett. 85, 182 (2000)

39. E. Il'ichev, F. Tafuri, M. Grajcar, R.P.J. IJsselsteijn, J. Weber, F. Lombardi, and J.R. Kirtley, Phys. Rev. B68, 04510 (2003).

40. G. Balestrino, S. Lavanga, P.G. Medaglia, S. Martellucci, A. Paoletti, G. Pasquini, G. Petrocelli, A. Tebano, A.A. Varlamov, L. Maritato, and M. Selvato, Phys. Rev. B62, 9835 (2000).

41. G. Balestrino, S. Lavanga, P.G. Medaglia, P. Orgiani, and A. Tebano, Phys. Rev. B64,020506 (2001).

42. F. Tafuri, J.R. Kirtley, P.G. Medaglia, P. Orgiani, and G. Balestrino, Phys. Rev. Lett. 92, 157006 (2004).

43. J. Pearl, J. Appl. Phys. 37, 4139 (1966).

44. V.G. Kogan, V.V. Dobrovitski, and J.R. Clem, Phys. Rev. B63, 144501 (2001). 